\title{
Spectra of subnormal Hardy type operators
}

\author{
by K. Rudol (Kraków)
}

\begin{abstract}
The essential spectrum of bundle shifts over Parreau-Widom domains is studied. Such shifts are models for subnormal operators of special (Hardy) type considered earlier in $[A D],[R 1]$ and [R2]. By relating a subnormal operator to the fiber of the maximal ideal space, an application to cluster values of bounded analytic functions is obtained.
\end{abstract}

Introduction. The study of Hardy space models for non-cyclic subnormal operators $S$ was initiated by Sarason (for operators related to simply connected domains $\Omega$ and annuli) and by Abrahamse and Douglas [AD] (in the case of finitely connected $\Omega$ ). They succeeded in representing $S$ (up to unitary equivalence) as an orthogonal sum of a normal operator and of a pure subnormal operator unitarily equivalent to the bundle shift $T_{E}$ for some flat unitary bundle $E$ spread over $\Omega$. The latter domain $\Omega$ was typically the interior of $\sigma(S)$ and $T_{E}$ was defined on the Hardy space $H^{2}[E]$ of certain cross sections $f: \Omega \rightarrow E$ in the simplest possible way: as multiplication by the independent variable,

$$
\left(T_{E} f\right)(\lambda)=\lambda f(\lambda), \quad \lambda \in \Omega .
$$

The essential assumptions were:

(1) the mutual position of the spectra of $S$ and of its minimal normal extension (it is here that $\Omega$ emerges),

(2) the sufficient regularity of $\Omega$.

Condition (1), stated below in (1.2), is of general nature and it can be used to distinguish between the two principal examples of pure subnormal operators: the Bergman space shifts and the Hardy space shifts (cf. [C]). This fact combined with the model theorem motivated our naming after Hardy of the relevant class of pure subnormal operators proposed in Definition 1.1 below. The regularity requirements (2) for the model theorem were subse-

1991 Mathematics Subject Classification: Primary 47B20; Secondary 46J15, 32E25. Key words and phrases: subnormal operators, Hardy classes, function algebras. 
quently relaxed (cf. [R1], [R2]), to include a wide class of infinitely connected domains.

In the present paper we investigate spectral properties of bundle shifts. This implies useful (and difficult to obtain directly) information for general Hardy type operators via their model theory. A special class of Hardy type operators related to fiber algebras is used in Section 2 to obtain a version of the Iversen theorem on cluster sets of bounded analytic functions.

1. Preliminaries. We consider subnormal operators $S$ on complex separable Hilbert spaces $H$. Subnormality means the existence of a normal operator $N$ (called a normal extension of $S$ ) on some space $K \supseteq H$, with $N(H) \subseteq H$ and $\left.N\right|_{H}=S$. Purity of $S$ means that none of its restrictions to nonzero invariant subspaces is normal. In what follows we assume $N$ to be a minimal normal extension of $S$. Then the special relations between their spectra (known as the Spectral Inclusion Theorem) hold:

$$
\partial \sigma(S) \subseteq \sigma(N) \subseteq \sigma(S) .
$$

We have, among others, the two extreme possibilities: one (e.g. Bergman shifts) when $\sigma(S)=\sigma(N)$, and the other when $\sigma(N)$ is only the boundary, $\partial \sigma(S)$ of the spectrum of $S$ (typical for Hardy shifts on $H^{2}(\Omega)$ ). A slightly modified version of this second possibility defines our class of operators as follows.

Definition 1.1. Let $\Omega$ be a domain in the complex plane $\mathbb{C}$. We say that a bounded operator $S$ is of Hardy type with respect to $\Omega$ if $S$ is pure subnormal and the spectra of $S$ and of its minimal normal extension $N$ satisfy the following condition:

$$
\sigma(S) \subseteq \bar{\Omega} \quad \text { and } \quad \sigma(N) \subseteq \partial \Omega .
$$

One easily deduces that such a set is necessarily bounded, with $\bar{\Omega}=\sigma(S)$ if only $\sigma(S) \cap \Omega \neq \emptyset$. Moreover, the pure part of a subnormal operator satisfying (1.2) is of Hardy type (cf. [R1, (2.1)]), which allows one to reduce the proof of the model theorem to the case when $S$ itself is pure. Note that the set $\Omega$ can be determined by $S$ satisfying (1.2) only under some regularity assumptions on $\Omega$. This was the case in the original setting of [AD], where $\partial \Omega$ was supposed to consist of finitely many analytic Jordan curves.

Let us now collect some necessary information from $[\mathrm{AD}],[\mathrm{W}]$ related to bundles. Recall that $E$ (more precisely, the pair $(E, \pi)$, where $\pi: E \rightarrow \Omega$ is a continuous mapping), is a flat unitary bundle over $\Omega$ if $E$ is a topological space, its fibers $E_{\lambda}=\pi^{-1}\{\lambda\}$ over the points $\lambda \in \Omega$ are Hilbert spaces and $\Omega$ has a covering $\{U: U \in \mathcal{W}\}$ by open sets $U$ such that $\pi^{-1} U=E_{U}$ are homeomorphic via some mappings $\tau_{U}$ to trivial bundles $U \times K_{U}$. Here $K_{U}$ are certain Hilbert spaces and $\tau_{U}: E_{U} \rightarrow U \times K_{U}$ are assumed to be 
compatible in such a way that the transition functions

$$
\tau_{U} \circ \tau_{V}^{-1}:(U \cap V) \times K_{V} \rightarrow(U \cap V) \times K_{U}
$$

map $(\lambda, x)$ into $\left(\lambda, \tau_{\lambda}^{U V}(x)\right)$ with $\tau_{\lambda}^{U V}: K_{V} \rightarrow K_{U}$ some unitary operators depending holomorphically on $\lambda \in U \cap V$. A mapping $f: \Omega \rightarrow E$ is called a holomorphic cross-section of $E$ if $\pi(f(\lambda))=\lambda(\forall \lambda \in \Omega)$ and $\left.\tau_{U} \circ f\right|_{U}$ : $U \rightarrow U \times K_{U}$ are holomorphic $(\forall U \in \mathcal{W})$. Note that for $\lambda \in \Omega$ the norms $\|f(\lambda)\|_{U}$ in the coordinate spaces $K_{U}$ are the same for all $U \in \mathcal{W}$ whenever $\lambda \in U$, so that the notation $\|f(\cdot)\|$ for the function $\Omega \ni \lambda \mapsto\|f(\lambda)\|$ is unambiguous.

Definition 1.3. For $1 \leq p<\infty$ the Hardy space $H^{p}[E]$ of a flat unitary bundle $E$ is the set of all holomorphic cross-sections $f$ of $E$ such that the function $\|f(\cdot)\|^{p}$ has a harmonic majorant, i.e. a harmonic function $h: \Omega \rightarrow[0, \infty)$ satisfying

$$
\|f(\lambda)\|^{p} \leq h(\lambda) \quad \forall \lambda \in \Omega .
$$

The norm $\|f\|_{p}$ of $f \in H^{p}[E]$ is defined with respect to some fixed norming point $\lambda_{0} \in \Omega$ as the quantity

$$
\|f\|_{p}=\left(h\left(\lambda_{0}\right)\right)^{1 / p},
$$

in which $h$ is the least harmonic majorant of $\|f(\cdot)\|^{p}$.

Another way of computing $\|f\|_{p}$ is to exhaust $\Omega$ by an increasing sequence of smoothly bordered finitely connected domains $\Omega_{n}$ such that $\lambda_{0} \in \Omega_{1}, \bar{\Omega}_{n} \subset \Omega_{n+1}$. With $\omega_{n}$ denoting the harmonic measure for $\Omega_{n}$ from (the same) point $\lambda_{0}$, one has

$$
\begin{aligned}
& \|f\|_{p}=\left(\lim _{n} \int_{\partial \Omega_{n}}\|f(\lambda)\|^{p} d \omega_{n}(\lambda)\right)^{1 / p}, \\
& \langle u, v\rangle=\lim _{n} \int_{\partial \Omega_{n}}\langle u(\lambda), v(\lambda)\rangle d \omega_{n}(\lambda)
\end{aligned}
$$

for any $f \in H^{p}[E]$ and $u, v \in H^{2}[E]$. In particular, if $E$ is a trivial bundle $\Omega \times K$ (so that $\pi(\lambda, k)=\lambda$ ), we identify its sections with functions $f: \Omega \rightarrow$ $K$, denoting the corresponding Hardy class by $H_{K}^{p}(\Omega)$. In the scalar-valued case $(K=\mathbb{C})$, we write $H^{p}(\Omega)$.

It may happen that for some bundle over $\Omega$ all Hardy classes are trivial: $H^{p}[E]=\{0\}$. From now on we assume that $\Omega$ is a Parreau-Widom $(\mathrm{P}-\mathrm{W})$ domain, i.e., $H^{1}[F] \neq\{0\}$ for all flat unitary bundles $F$ over $\Omega$. A necessary and sufficient condition is formulated in [W] in terms of the Green function $g(\cdot, \zeta)$ with pole at a (fixed) point $\zeta \in \Omega$ : For $\alpha>0$ let $B(\alpha)$ be the first 
Betti number of the set $\{z \in \Omega: g(z, \zeta)>\alpha\}$. Then $\Omega$ is a $\mathrm{P}-\mathrm{W}$ domain iff

$$
\int_{0}^{\infty} B(\alpha) d \alpha<\infty
$$

In the latter case, even more is true: the extremal methods of [W] yield the possibility of constructing $H^{\infty}[E]$-sections $f$ with arbitrarily prescribed value $f\left(\lambda_{1}\right)$ at a fixed point $\lambda_{1}$ of $\Omega$.

Now we can start analysing spectra of the bundle shift operator $T_{E}$ defined by (1.0) on $H^{p}[E]$, with special attention to the Hilbert space case $p=2$. One immediate, but useful, observation is that these spaces are $H^{\infty}(\Omega)$-modules: $\lambda \mapsto \varphi(\lambda) f(\lambda)$ belongs to $H^{p}[E]$ whenever $f$ does and $\varphi \in$ $H^{\infty}(\Omega)$. Our further considerations require assuming the $(\mathrm{P}-\mathrm{W})$ condition on $\Omega$.

Let us make the following observation.

Lemma 1.5. For $\lambda_{1} \in \Omega$ the range $R\left(T_{E}-\lambda_{1}\right)$ equals $\left\{h \in H^{p}[E]\right.$ : $\left.h\left(\lambda_{1}\right)=0\right\}$ and its codimension is equal to $\operatorname{dim} E_{\lambda_{1}}$.

Proof. Let us fix some $H^{\infty}[E]$-sections $f_{j}, j \leq \operatorname{dim} E_{\lambda_{1}}$, so that $\left\{f_{j}\left(\lambda_{1}\right)\right\}$ form a basis of $E_{\lambda_{1}}$. Take any $f \in H^{p}[E]$. Then with proper choice of the scalars $\alpha_{j}$, one has $f\left(\lambda_{1}\right)=\sum_{j} \alpha_{j} f_{j}\left(\lambda_{1}\right)$ and $h=f-\sum_{j} \alpha_{j} f_{j} \in$ $H^{p}[E]$ satisfies $h\left(\lambda_{1}\right)=0$. Using local coordinates one verifies that $g(\lambda)=$ $\left(\lambda-\lambda_{1}\right)^{-1} h(\lambda)$ has a removable singularity at $\lambda_{1}$, while a standard distance estimate and subharmonicity of $\|g(\cdot)\|^{p}$ imply $g \in H^{p}[E]$. Clearly, $h=\left(T_{E}-\lambda_{1}\right) g$. Hence the $f_{j}$ form a linear basis of the complement of $R\left(T_{E}-\lambda_{1}\right)$.

Proposition 1.6. $T_{E}$ is a bounded linear operator on $H^{p}[E]$, with $\sigma\left(T_{E}\right)$ $=\bar{\Omega}$. For the interior points $\lambda \in \Omega, T_{E}-\lambda$ is semi-Fredholm with index equal to $-\operatorname{dim} E_{\lambda}(=-\operatorname{rank}(E))$. Hence the essential spectrum coincides with the spectrum if $E$ has infinite rank, and is contained in $\partial \Omega$ in the finite rank case. For $p=2, T_{E}$ is a subnormal operator of Hardy type.

Note that since $\partial \sigma(T) \subseteq \sigma_{\mathrm{e}}(T)$, this determines also the essential spectrum except for inner boundary points.

Proof. In view of Lemma 1.5, only the subnormality requires verification. Choose an increasing exhaustion of $\Omega$ with smoothly bordered domains $\Omega_{n}$. The corresponding limits (1.4) of inner products yield the Halmos-Bram subnormality condition (cf. [C]). The purity can be shown in a similar manner to the proof of Prop. 2.6(2) (an argument involving analytic structure and order of zeros) in the next section. To characterize the spectrum of a normal extension of $T_{E}$ one can follow the procedure of lifting functions (or sections) on $\Omega$ to the unit disc via the universal covering map $\varrho$ for $\Omega$. $T_{E}$ is unitarily equivalent to multiplication by $\varrho$ on a subspace of vector-valued 
$L^{2}$-type functions on the unit circle. The essential range of $\varrho$ is the spectrum of a normal extension and is contained in $\partial \Omega$, by a purely topological argument (cf. [R1]). Note that the claim on Hardy type of $T_{E}$ uses the $(\mathrm{P}-\mathrm{W})$ condition only to rule out the possibility of $H^{2}[E]=\{0\}$.

2. Iversen's theorem and subnormal operators. In this section we consider one interesting example of subnormal Hardy type operators and apply it to classical function theory. We shall obtain a convenient perspective on problems concerning cluster sets of bounded analytic functions (also in several complex variables). This idea came as a result of attempts to localize the author's earlier result on behaviour of "global cluster sets".

Let $\mathcal{G}$ be a bounded domain in $\mathbb{C}^{n}$. Given $\xi \in \mathbb{C}^{n}$ we denote by $\mathcal{M}_{\xi}(\mathcal{G})$ (or briefly $\mathcal{M}_{\xi}$ ) the fiber over $\xi$ of the maximal ideal space, $\mathcal{M}(\mathcal{G})$, of the Banach algebra $H^{\infty}(\mathcal{G})$ of bounded holomorphic functions on $\mathcal{G}$ (cf. [G1]). In other words, $\mathcal{M}_{\xi}(\mathcal{G})$ is the preimage of $\{\xi\}$ under the $n$-tuple $\widehat{Z}$ of Gelfand transforms of coordinate functions $Z=\left(z_{1}, \ldots, z_{n}\right)$. The fibers are related (for "regular" domains $\mathcal{G}$ ) to cluster sets,

$$
\mathrm{Cl}(h ; \xi)=\left\{\lim h\left(\xi_{n}\right): \xi_{n} \in \mathcal{G}, \xi=\lim \xi_{n}\right\} .
$$

The well-known (at least in the $n=1$ case) Cluster Value Theorem states that the fibers over points outside $\overline{\mathcal{G}}$ are empty, while for $\xi \in \overline{\mathcal{G}}$ and $h \in$ $H^{\infty}(\mathcal{G})$ one has

$$
\widehat{h} \mathcal{M}_{\xi}(\mathcal{G})=\mathrm{Cl}(h ; \xi) .
$$

Various sufficient regularity conditions were given by many authors (e.g. by T. W. Gamelin - that $\mathcal{G}$ be a polydomain, i.e. the cartesian product of arbitrary bounded plane domains, and by I. Graham for the unit ball). Arguments in the strictly pseudoconvex case were compiled in $[\mathrm{Cu}]$ and $[\mathrm{R}]$, and extended in [R0] (where precise references can be found) to any domain $L^{\infty}$-[strongly] regular for the $\bar{\partial}$ operator. This regularity means that for any $\bar{\partial}$-closed $(0, p)$-form $\varphi=\sum \varphi_{\alpha} d \bar{z}_{\alpha}$ with bounded [resp. continuous on $\overline{\mathcal{G}}$ ] coefficients $\varphi_{\alpha}$ the equation $\bar{\partial} \psi=\varphi$ has a solution among $(0, p-1)$-forms $\psi$ with bounded [resp. continuous] coefficients $\psi_{\beta}$.

The continuity part is required for constructing the peak function needed in establishing a uniqueness claim and if this is available directly, then the continuity requirements (i.e. the strong regularity) can be reduced to the $L^{\infty}$-regularity alone. The latter holds for any analytic polyhedra and for spsc. domains with nonsmooth boundaries. The only known examples of domains without the Cluster Value Property seem to be N. Sibony's counterexamples to the Corona Theorem in two complex variables, where some fibers over points $t \notin \overline{\mathcal{G}}$ are nonempty, but the author knows no example of (2.1) failing at a boundary point. 
In what follows we additionally assume that $\xi$ is an essential boundary point (i.e. not all functions from $H^{\infty}(\mathcal{G})$ extend analytically to a neighbourhood of $\xi$ in $\left.\mathbb{C}^{n}\right)$. Otherwise, the cluster sets are singletons, not interesting from our point of view.

We refer to $[\mathrm{G}]$ for standard terminology related to function algebras. In particular, we treat $H^{\infty}(\mathcal{G})$ as a function algebra $A$ on $\mathcal{M}=\mathcal{M}(\mathcal{G})$. The Choquet boundary of $\operatorname{Re} A$ is the set

$$
\mathrm{P}-\mathrm{P}(A)
$$

of p-points, or generalized peak points $\gamma$ of $A$, characterised by the existence for any fixed neighbourhood $U$ of $\gamma$ and $1>\alpha>0$ of a function $\varphi \in A$ satisfying

$$
|\varphi| \leq \alpha \quad \text { on } \mathcal{M} \backslash U, \quad|\varphi|>1 \quad \text { on } U .
$$

By the classical Bishop-de Leeuw theorem [G, Chapter 12], every point of $\mathcal{M}$ has a representing measure $\nu$ concentrated on the set $\mathrm{P}-\mathrm{P}(A)$ of all $p$-points of $A$ (i.e. for any Borel set $Y \subset \mathcal{M} \backslash(\mathrm{P}-\mathrm{P}(A))$ one has $\nu(Y)=0)$.

The fiber algebra (denoted here for brevity by $A_{\xi}$ ) is the restriction of $A$ to the fiber $\mathcal{M}_{\xi}$, i.e.

$$
A_{\xi}=\left\{\left.\widehat{h}\right|_{\mathcal{M}_{\xi}}: h \in H^{\infty}(\mathcal{G})\right\} .
$$

From now on we fix an essential boundary point $\xi$, imposing moreover the following regularity condition.

Assumption 2.3. Assume that any $p$-point in the maximal ideal space of $A_{\xi}$ is also a $p$-point with respect to $A$ (i.e. $\left.\mathrm{P}-\mathrm{P}\left(A_{\xi}\right) \subset \mathrm{P}-\mathrm{P}(A)\right)$. Moreover, assume that the Cluster Value Theorem holds in $\mathcal{G}$.

The first condition follows from Cor. 12.9 of $[\mathrm{G}]$, if $\mathcal{M}_{\xi}$ is an intersection of peak sets for $A$. This takes place if there exists a continuous function $g$ on $\overline{\mathcal{G}}$, analytic on $\mathcal{G}$ that peaks at $\xi:$ for $\zeta \in \overline{\mathcal{G}} \backslash\{\xi\}$ one has $|g(\zeta)|<1$, while $g(\xi)=1$. The first assumption holds not only at strongly pseudoconvex points $\xi$, but also at boundary points of polydomains [G1]. It should be remarked that in the latter case one can reduce the study of fiber algebras to the case where $\xi$ belongs to the distinguished boundary, via canonical isomorphisms to "lower-dimensional fibers" constructed in [G1].

In what follows we fix an essential boundary point $\xi$ and a bounded analytic function $h$ on $\mathcal{G}$ such that $\mathrm{Cl}(h ; \xi)$ contains an interior (in the Euclidean topology) point, say $\zeta$. By Assumption 2.3, $\zeta=\widehat{h}(\tau)$ for some $\tau \in \mathcal{M}_{\xi}$. We can further fix a representing measure $\eta$ for this $\tau$ concentrated in the above sense on the set of $p$-points for $A_{\xi}$. Note that the fiber algebra consists of continuous functions on the compact space $\mathcal{M}_{\xi}$, hence it is contained in $L^{2}(\eta)$ and one can consider its closure $H^{2}\left(A_{\xi}, \eta\right)$ in the $L^{2}(\eta)$ norm topology, usually called the abstract Hardy space. 
Definition 2.4. The fiber multiplication operator $T=T_{h}^{(\xi, \eta)}$ is defined on $H^{2}\left(A_{\xi}, \eta\right)$ by

$$
\left(T_{h}^{(\xi, \eta)} f\right)(x)=\widehat{h}(x) f(x) \quad \text { for }[\eta] \text { a.e. } x \in \mathcal{M}_{\xi}, f \in H^{2}\left(A_{\xi}, \eta\right) .
$$

LEMma 2.5. $T$ is a bounded subnormal operator with $\sigma(T) \subseteq \mathrm{Cl}(h ; \xi)$, whose minimal normal extension has spectrum contained in the closure of $\widehat{h}\left(\mathrm{P}-\mathrm{P}\left(A_{\xi}\right)\right)$.

Proof. By the assumed cluster value property, the spectrum $\Sigma$ of $h$ with respect to the fiber algebra (of continuous functions on $\mathcal{M}_{\xi}$ ) equals $\mathrm{Cl}(h ; \xi)$. If $\lambda \notin \Sigma$, the fiber multiplication by the inverse in $A_{\xi}$ of $\widehat{h}-\lambda$ is equal on $\mathcal{M}_{\xi}$ to $\widehat{g}$ for some $g \in A$ and the fiber multiplication by $g$ is clearly $(T-\lambda)^{-1}$. The remaining claim follows from standard properties of multiplications defined on $L^{2}(\eta)$.

The purity of $T$ is a more delicate question and we prove it along with the Hardy type condition, although it holds under some weaker hypotheses.

Proposition 2.6. Assume that there exists a bounded plane domain $\Omega$ such that $\mathrm{Cl}(h, \xi) \subseteq \bar{\Omega}$ and $\widehat{h}\left(\mathrm{P}-\mathrm{P}\left(A_{\xi}\right)\right) \subseteq \partial \Omega$. Then the pure part $T_{\text {pur }}$ of $T$ is a Hardy type operator with respect to $\Omega$. Consequently, $\sigma(T)=\bar{\Omega}$. Only one of the following two possibilities may occur:

(1) $T$ has nonempty point spectrum and $\widehat{h}$ is constant on some subset of positive $[\eta]$ measure,

(2) $T$ is pure subnormal $\left(T_{\text {pur }}=T\right)$.

The second (most desirable) case takes place if one can embed an analytic disc around $\tau$ in the fiber, i.e. a family $\left\{\tau_{z}: z \in \mathbb{C},|z|<1\right\} \subset \mathcal{M}_{\xi}$ such that $\tau_{0}=\tau$ and the functions $z \mapsto \widehat{g}\left(\tau_{z}\right)$ are holomorphic in $z$ for any $g \in A$.

Proof of Proposition 2.6. In view of Lemma 2.5 and the remark following 1.1, the only nontrivial assertion to prove concerns the purity of $T$. Assume that for some closed nonzero subspace $W$ of $H^{2}\left(A_{\xi}, \eta\right)$ invariant under $T$, the restriction $\left.T\right|_{W}$ is a normal operator. Then $\left.T\right|_{W}$ is a direct orthogonal summand of any normal extension of $T$ and the assumptions imply $\sigma\left(\left.T\right|_{W}\right) \subseteq \partial \Omega$. By our earlier assumption, $\mathrm{Cl}(h ; \xi)$ contains an interior point, namely $\zeta=\widehat{h}(\tau)$. Replacing $T$ with $T-\zeta$ if necessary, we may assume $\zeta=0$. Hence $0 \in \Omega, 0 \notin \partial \Omega$ and this implies the invertibility of $\left.T\right|_{W}$. Now $W=T(W)$ implies

$$
W \subseteq\left\{f \in H^{2}\left(A_{\xi}, \eta\right): \int f d \eta=0\right\} .
$$

The diagonalisation procedure for multiplication operators (cf. $[\mathrm{AK}]$ ) yields equivalence of $W$ to a reducing subspace of the form $\int^{\oplus} K_{z} d \kappa$, where $\kappa$ is the image of $\eta$ under $\widehat{h}$, while $K_{z}$ are subspaces of $L^{2}\left(\eta_{z}\right)$ for $\eta_{z}$ obtained by 
disintegration of $\eta$ with respect to $\widehat{h}$. The element of $L^{2}\left(\eta_{z}\right)$ corresponding to $f$ is simply the equivalence class of $f$ modulo equality a.e. $\left[\eta_{z}\right]$. From (2.7) we get

$$
\int\left(\int f d \eta_{z}\right) d \kappa(z)=0, \quad f \in W
$$

but the latter can occur for all elements of $\int^{\oplus} K_{z}$ only when the direct integral decomposition is trivial (reduces to $W=K_{z^{0}}$ for one point $z^{0}$, an atom of $d \kappa)$. Hence $T$ acts on $W$ as a scalar multiple of the identity, with $z^{0} \in \sigma_{\mathrm{p}}(T)$.

If the $\tau_{z}$ form an analytic disc around $\tau$, then $W=T W\left(=T^{2} W=\ldots\right)$, $\widehat{h}(\tau)=0$ implies that for any nonzero $f \in W$ the analytic function $z \mapsto \widehat{f}\left(\tau_{z}\right)$ is divisible by $z \mapsto\left(\widehat{h}\left(\tau_{z}\right)\right)^{k}, k=1,2, \ldots$, hence has a zero of infinite order, which is impossible.

Assumption 2.8. Let $\mu$ be a measure on $\mathcal{M}$ representing evaluation at a point of $\mathcal{G}$ for $A=H^{\infty}(\mathcal{G})$ such that any other point from a fixed dense subset of $\mathcal{G}$ has a representing measure with support contained in $\operatorname{supp}(\mu)$.

Well-known results on abstract function algebras [G] imply that such a $\mu$ always exists. In the case of plane domains or polydomains, the harmonic measure's lift to the Shilov boundary satisfies 2.8 (cf. [G1]). So do the measures implicit in integral formulae for functions in terms of certain boundary values (cf. remarks that follow 2.10 below). The standard proof of the following fact is omitted.

Lemma 2.9. If $\mu$ satisfies the above assumptions, then its closed support must contain $\mathrm{P}-\mathrm{P}(A)$.

Definition. The $\mu$-asymptotic cluster set $\mathrm{Cl}^{\mu}(h ; \xi)$ of $h \in H^{\infty}(\mathcal{G})$ at $\xi \in \partial \mathcal{G}$ is the intersection over open sets $U \supset \mathcal{M}_{\xi}$ of $\mu_{U}$-essential images of $\widehat{h}$. Here $\mu_{U}$ is the restriction of $\mu$ to $U$.

In the case of lifted harmonic measure, these sets are nicely described (cf. [G1]) in terms of behaviour of $h$ at certain curves approaching $\partial \mathcal{G}$. Here (and for the mentioned measures related to integral formulae) the asymptotic values at "measure zero sets" are negligeable. All such cluster sets are contained in the standard asymptotic cluster set. The following variant of Iversen's theorem is now a consequence of the Spectral Inclusion Theorem for subnormal operators.

Theorem 2.10. Assuming (2.3) and (2.9) we have

$$
\partial \mathrm{Cl}(h ; \xi) \subseteq \mathrm{Cl}^{\mu}(h ; \xi) .
$$

Proof. The left-hand side is $\partial \sigma(T)$, and hence is contained in the $\eta$ essential image of $\widehat{h}$, which, by continuity, is contained in the closure of $\widehat{h}\left(\mathrm{P}-\mathrm{P}\left(A_{\xi}\right)\right)$. Now the result becomes a topological consequence of $(2.3)$. 
Remark 1. The corresponding result in [G1] referred to the Shilov boundary and required more delicate analysis. The methods were applicable to a narrower class of domains (polydomains). Note also that by composing $h$ with an appropriate fractional linear function $\phi$ one extends the above Iversen's theorem to the case when $h$ is meromorphic.

Remark 2. The set $\mathrm{Cl}^{\mu}(h ; \xi)$ can be defined in a special geometric manner mentioned above. Namely, assume that there exists a measure $\mu$ on $\partial \mathcal{G}$ and a collection of curves $\ell_{z}, z \in \partial \mathcal{G}$, in $\mathcal{G}$ terminating at $z$ such that any bounded holomorphic function $h$ on $\mathcal{G}$ has boundary limits along $\ell_{z}$ for $[\mu]$ almost every $z \in \partial \mathcal{G}$, and the values $h\left(z_{0}\right)$ at any interior point $z_{0}$ are integrals of these boundary values for appropriate representing measures on $\partial \mathcal{G}$, absolutely continuous with respect to $\mu$. Then appropriate limit points of the $\ell_{z}$ can be used as points supporting the representing measures mentioned in 2.8 on the maximal ideal space $\mathcal{M}$. $\mathrm{As}^{\mu} l^{\mu}(h ; \xi)$ we can take the asymptotic cluster set defined by the asymptotic boundary values along $\ell_{z}, z \in U \cap \partial \mathcal{G}$.

\section{References}

[AD] M. B. Abrahamse and R. G. Douglas, A class of subnormal operators related to multiply connected domains, Adv. in Math. 19 (1976), 106-148.

[AK] M. B. Abrahamse and T. Kriete, The spectral multiplicity of a multiplication operator, Indiana Univ. Math. J. 22 (1973), 845-857.

[C] J. B. Conway, Subnormal Operators, Pitman, Boston, 1981.

[C1] - Spectral properties of certain operators on Hardy spaces of planar domains, Integral Equations Operator Theory 10 (1987), 659-706.

[Cu] J. Cufi, $H^{\infty}+C$ in several variables, Collect. Math. 23 (1982), 109-123.

[G] T. W. Gamelin, Uniform Algebras, Prentice-Hall, Englewood Cliffs, N.J., 1969.

[G1] - Iversen's Theorem and fiber algebras, Pacific J. Math. 46 (1973), 389-414.

[M] W. Mlak, Szegő measures related to plane sets, Comment. Math., Tomus spec. in honorem L. Orlicz 1 (1978), 239-249.

[R] K. Rudol, On spectral mapping theorems, J. Math. Anal. Appl. 97 (1983), 131139.

[R0] - Spectral mapping theorems for analytic functional calculi, in: Adv. in Invariant Subspaces and Other Results of Operator Theory, R. G. Douglas et al. (eds.), Oper. Theory: Adv. Appl. 17, Birkhäuser, 1986, 331-340.

[R1] - , The generalised Wold Decomposition for subnormal operators, Integral Equations Operator Theory 11 (1988), 420-436.

[R2] - Subnormal operators of Hardy type, in: Banach Center Publ. 38, Inst. Math., Polish Acad. Sci., 1997, 315-324.

[S] M. V. Samokhin, Some classical problems of analytic functions theory in Parreau-Widom domains, Mat. Sb. 182 (1991), 892-910 (in Russian).

[S1] - On limit properties of bounded holomorphic functions and maximum modulus principle in domains of arbitrary connectivity, ibid. 135 (1988), 497-513 (in Russian). 
[W] H. Widom, $H^{p}$ sections of vector bundles over Riemann surfaces, Ann. of Math. 94 (1971), 304-324.

Institute of Mathematics

Polish Academy of Sciences

Św. Tomasza 30

31-207 Kraków, Poland

E-mail: narudol@cyf-kr.edu.pl

Reçu par la Rédaction le 3.1.1996

Révisé le 10.4.1996 